Revista de Psicología de la PUCP. Vol. XXI, 1, 2003 Edición de aniversario

\title{
Psicología de la salud y adicciones: perspectiva terapéutica
}

\author{
Elisardo Becoña Iglesias ${ }^{1}$ \\ Universidad de Santiago de Compostela, España \\ Luis Armando Oblitas Guadalupe ${ }^{2}$ \\ Universidad Iberoamericana, México
}

\begin{abstract}
El tema de las adicciones sigue tan vigente como en el siglo pasado, no solo por el incremento del número de personas que lo padecen sino también por las importantes secuelas que produce en el individuo y en su entorno. Existen diferentes enfoques teóricos para abordar la problemática de las adicciones, sin embargo el que nos parece más conveniente por sus resultados terapéuticos es el que proviene desde la Psicología de la salud, basado en un enfoque integral en el abordaje del sujeto. El artículo fundamentalmente describe un esquema terapéutico general de su aplicación a los adictos, desde el enfoque cognitivo-conductual.

Palabras clave: adicciones, tratamiento, salud, técnicas, perspectivas.
\end{abstract}

The psychology of health and addictions: therapeutic perspective

The addiction subject is nowadays a valid one, as well as in the past century. Not only because of the increase of people that are addict, but also because of the important effects that cause on people and their environments. There are many theoretical perspectives to approach the addiction problem, but the most convenient because of its therapeutic results is the one that is supported by the psychology of health. It is based on the integral approach to the person. This paper describes a general therapeutic scheme to work with addicts from the cognitivebehavioral perspective.

Key words: Addiction, treatment, health, technics, perspectives.

1 Doctor en Psicología. Es catedrático de Psicología Clínica (Técnicas de Modificación de Conducta) en el Departamento de Psicología Clínica y Psicobiología de la Facultad de Psicología de la Universidad de Santiago de Compostela. Codirige desde el curso de postgrado "Master en Drogodependencias de la Universidad de Santiago de Compostela" y el "Programa Interuniversitario de Formación de Especialistas en Tabaquismo". Es el responsable de la Unidad de Tabaquismo donde se aplica el Programa para dejar de fumar que desde el año 1984 en la Universidad de Santiago de Compostela.

2 Doctor en Psicología por la Universidad Nacional Autónoma de México y Magíster en Psicología por la Universidad Santo Tomás de Aquino (Colombia). Es consultor académico del Instituto Mexicano de la Pareja, del Instituto Mexicano de Psicoterapia Guestalt, del Instituto Mexicano de Terapia Cognitivo Conductual y de la Asociación Mexicana para la Intervención en Crisis. Asimismo, es fundador y editor de la Revista Internacional de Psicología. Actualmente imparte el seminario de Psicología de la salud en la UNAM Iztacala y en la Ibero Puebla, y el seminario de investigación en la Maestría en Psicoterapia Guestalt. Suele 



\section{Antecedentes}

La historia de las adicciones va unida a la historia del hombre. Fumar cigarrillos, beber alcohol, mascar hojas de coca, esnifar preparados psicoactivos, beber pócimas, fumar marihuana, utilizar el opio para el dolor, etc., son ejemplos bien conocidos de algunas de las sustancias que el hombre ha utilizado o sigue utilizando a lo largo de la historia. Más actualmente, junto a las anteriores, y sus derivados industriales o químicos, destacan las nuevas adicciones, unas derivadas de sustancias, como es el caso de la heroína, la cocaína, las drogas de diseño, el LSD, entre las más importantes, y otras adicciones comportamentales, sin sustancia, como resultado de nuestra sociedad tecnológica, como la adicción a Internet, al juego de azar, al teléfono móvil, a los teléfonos eróticos, al sexo, a las compras y a un amplio etcétera de conductas que pueden llegar a ser adictivas (Becoña, 1998b).

Por ello, en los últimos años se incluyen distintas conductas bajo la denominación genérica de adicciones o conductas adictivas. Basadas inicialmente en el concepto de dependencia (física y psíquica), y evolucionando a partir del mismo, se aplicaban inicialmente a sustancias psicoactivas que ingeridas por un individuo tenían la potencialidad de producir dependencia. Con el transcurrir de los años se observó que también existían conductas, que sin haber sustancia de por medio, tenían la capacidad de producir dependencia y el resto de las características que tenían las dependencias a las sustancias psicoactivas.

dictar seminarios en países como El Salvador, Perú, Colombia y España. Av. de las Fuentes 41-A, piso 1. Lomas de Tecamachalco, CP 53850. Estado de México. Correo electrónico: oblitas@thomsonlearning.com.mx. 
Una característica central a las conductas adictivas es la pérdida de control. La persona con una conducta adictiva no tiene control sobre esa conducta, aparte de que la misma le produce dependencia, tolerancia, síndrome de abstinencia y una incidencia negativa muy importante en su vida, que va a ser en muchos casos la causa de que acuda en busca de tratamiento o la obliguen a buscarlo. Gossop (1989) definió como elementos característicos de una adicción: (a) un fuerte deseo o un sentimiento de compulsión para llevar a cabo la conducta particular (especialmente cuando la oportunidad de llevar a cabo tal conducta no está disponible); (b) la capacidad deteriorada para controlar la conducta (especialmente, en términos de controlar su comienzo, mantenimiento o nivel en el que ocurre); (c) malestar y estado de ánimo alterado cuando la conducta es impedida o la deja de hacer; y (d) persistir en la conducta a pesar de la clara evidencia de que le está produciendo graves consecuencias al individuo. En la misma línea, Echeburúa (1999) considera como características princjpales de las conductas adictivas la pérdida de control, la fuerte dependencia psicológica, la pérdida de interés por otras actividades gratificantes y la interferencia grave en la vida cotidiana.

Si tuviésemos que hablar de diferencias entre las adicciones del pasado y las actuales, nosotros consideramos que hay tres diferencias fundamentales entre unas y otras. Estas son: (a) la disponibilidad y comercialización (sea de tipo legal o ilegal) a lo largo de todo el planeta de sustancias o productos que producen adicciones en los individuos, (b) la pérdida del sentido simbólico y del valor cultural que tenían en el pasado muchas de las adicciones actuales, que en aquel contexto se consumían controladamente de un modo normativizado y ritualizado, y (c) el cambio social que facilita el individualismo, la búsqueda del placer inmediato y la satisfacción de todas las necesidades que piensa el individuo que le son imprescindibles, facilitando con ello caer más fácilmente en las adicciones.

Respecto a la primera, hoy es indudable que la disponibilidad de sustancias y conductas con poder adictivo es enorme. Además, las 
Psicología de la salud y adiciones: perspectiva terapéutica

sustancias se han miniaturizado, en el sentido comercial y físico, consiguiéndose con ello una posibilidad de transporte fácil de las mismas y casi ilimitado en cantidad (Westermeyer, 1998). Por ejemplo no es lo mismo el número de dosis para el consumo que obtenemos de una tonelada de hoja de coca que de una tonelada de cocaína. La segunda permite multiplicar el número de dosis para el consumo por varias cifras respecto a la primera.

La pérdida del sentido simbólico de muchas sustancias ha facilitado un incremento de las adicciones. En muchas culturas la sustancia o la conducta tenía un valor simbólico o ritual, y como tal valor estaba sometida a las normas y al control social. Sin ese control social, en muchos casos precisamente para evitar excesos, el exceso ritual pasa a convertirse en un exceso frecuente. Esto lleva a que una parte de los individuos de ese sistema social tenga problemas con esa sustancia.

$\mathrm{Y}$, finalmente, los cambios sociales, económicos, tecnológicos y de todo tipo que hemos vivido en los últimos 50 años, han facilitado el cambio de un tipo de hombre a otro, en el más amplio sentido. Desaparece la ruralización y se incrementa la urbanización, se cambia el modo de producción, de intercambio de bienes, el modelo económico, produciendo falta de referentes en muchos individuos, etc. En otros casos, ante la disponibilidad de dinero que permite adquirir bienes, algunos optarán por la adquisición de aquellos más inmediatos que producen ese placer inmediato. Y, en este placer inmediato, están la mayoría de las adicciones.

El mayor problema que tienen las adicciones no son habitualmente los efectos que producen a corto plazo. El problema está en los efectos que producen a mediano y a largo plazo. Así, muchos fumadores de cigarrillos morirán años después de fumar ininterrumpidamente de cáncer de pulmón o de enfermedades cardiovasculares (Becoña y Vázquez, 1998); muchos bebedores excesivos de alcohol o 
alcohólicos morirán de enfermedades hepáticas o de accidentes; muchas personas dependientes de la heroína o de la cocaína morirán de enfermedades causadas por ellas, como ha ocurrido y está ocurriendo con el sida, la hepatitis, infecciones, etc., aparte de los problemas sociales que causan en forma de robo, extorsión, problemas legales, familiares, etc. Lo mismo podemos decir de las otras adicciones, donde en muchos casos la ruina económica es un paso previo al resto de los problemas legales, familiares, físicos, etc.

\section{Estado actual}

Hoy el primer elemento relevante en las adicciones es delimitar qué entendemos que es una adicción. A pesar de que hay criterios específicos para los distintos trastornos, como la dependencia de sustancias psicoactivas, el juego patológico, etc., todos parten de los criterios de dependencia de sustancias psicoactivas, dado que además en las adicciones, sean con o sin sustancia, se dan los fenómenos de pérdida de control, tolerancia, síndrome de abstinencia, etc. En esta línea, para el DSM-IV (American Psychiatric Association, 1994) la dependencia de sustancias psicoactivas se caracteriza por un patrón desadaptativo de consumo de la sustancia que conlleva un deterioro o malestar clínicamente significativos, expresado por tres o más de los síntomas que indicamos a continuación, y durante un período continuado de 12 meses. Estos síntomas son los siguientes:

1. Tolerancia, definida por cualquier de los siguientes ítems: (a) una necesidad de cantidades marcadamente crecientes de la sustancia para conseguir la intoxicación o el efecto deseado; (b) el efecto de las mismas cantidades de sustancia disminuye claramente con su consumo continuado.

2. Abstinencia, definida por cualquier de los siguientes ítems: (a) el síndrome de abstinencia característico para la sustancia; (b) se toma la misma sustancia (o una muy parecida) para aliviar o evitar los síntomas de abstinencia. 
3. La sustancia es tomada con frecuencia en cantidades mayores o durante un período más largo de lo que inicialmente se pretendía.

4. Existe un deseo persistente o esfuerzos infructuosos de controlar o interrumpir el consumo de la sustancia.

5. Se emplea mucho tiempo en actividades relacionadas con la obtención de la sustancia (por ej., visitar a varios médicos o desplazarse largas distancias), en el consumo de la sustancia (por ej., fumar un pitillo tras otro) o en la recuperación de los efectos de la sustancia.

6. Reducción de importantes actividades sociales, laborales o recreativas, debido al consumo de la sustancia.

7. Se continúa tomando la sustancia a pesar de tener conciencia de problemas psicológicos o físicos recidivantes o persistentes, que parecen causados o exacerbados por el consumo (por ej., consumo de la cocaína a pesar de saber que provoca depresión, o continuada ingesta de alcohol a pesar de que empeora una úlcera).

El DSM-IV también considera el abuso de sustancias que es una condición menos grave que la dependencia. En el caso de la dependencia de sustancias, cuando nos referimos a conductas sin sustancia, el diagnóstico sería el mismo sustituyendo sencillamente la palabra sustancia por la «específica» conducta adictiva (ej., juego, sexo, etc.), con los matices para esa específica conducta (Echeburúa, 1999).

\section{Técnicas de tratamiento para las adicciones y aplicaciones}

Objetivos a conseguir en el tratamiento de una conducta adictiva

Son muchas las distintas sustancias y conductas a las que las personas pueden adquirir dependencia. En cualquier intervención terapéutica con una persona con una adicción hay varios objetivos a conseguir en el proceso de tratamiento. Estos van graduados en función de la propia dependencia; esto es, poco se puede hacer con una intervención psicológica si la persona está intoxicada cuando acude a consulta; $o$, poco se puede hacer para intentar que mejore su estilo de vida saludable si no conoce medidas adecuadas para hacerlo. 
El primer objetivo del tratamiento es que la persona con una adicción asuma que necesita el tratamiento. Tanto en las drogas legales como en las ilegales hay un proceso de negación de muchos de los adictos a las mismas (Becoña, 1998a). La drogas les producen placer, bienestar, satisfacción (reforzamiento positivo) y, al mismo tiempo, la propia droga les permite evitar el síndrome de abstinencia (reforzamiento negativo) cuando no consumen o no tienen suficiente dosis. Por ello, la dependencia se mantiene en el tiempo y el proceso de reforzamiento hace que las graves consecuencias que suelen acompañar a éste impidan al principio asumir el problema. Asimismo, por los procesos de cambio, se sabe que la persona tiene que pasar por distintas fases antes de asumir que tiene que hacer un cambio.

Una vez que la persona ha asumido que tiene que cambiar va a solicitar distintos tipos de ayuda. Y es entonces de gran importancia hacerle ver claramente que precisa ayuda, que tiene que cambiar su conducta por las graves y evidentes consecuencias que le están produciendo, pero que la persona no ve en ese momento (por ejemplo, que to han despedido del trabajo, que ha tenido que abandonar los estudios, que tiene conflictos familiares, que to ha detenido la policía, que tiene que ingresar a prisión, que sus relaciones sociales han cambiado y ahora se relaciona sólo con amigos consumidores, etc.).

Superada la fase de negación de que el consumo de drogas es un problema o la minimización del mismo, hay que delimitar claramente por qué acude a pedir ayuda, factores relacionados y otras dificultades que ha causado la dependencia. Esta es la fase de evaluación.

En el tratamiento, éste se va a orientar inicialmente a que la persona consiga la abstinencia. Aquí va a variar el abordaje según se trate de una persona dependiente del alcohol, la nicotina, la heroína, cocaína o el juego o internet, pero dentro de más aspectos comunes que distintos en lo que atañe a las técnicas de tratamiento que utilizaremos con unos o con otros. Hoy existen, especialmente en el trata- 
Psicología de la salud y adiciones: perspectiva terapéutica

miento de la dependencia de la heroína, junto a los programas orientados a la abstinencia también los programas de reducción de daños, fundamentalmente los de mantenimiento de metadona.

En el caso de la heroína la desintoxicación cobra una gran relevancia, por estar presente en las personas dependientes a la misma tanto la dependencia física como la psicológica. Para la primera, se desintoxica al organismo mediante una interrupción brusca o paulatina de la sustancia. En la cocaína y drogas de síntesis, como en el juego patológico y casi todas las adicciones comportamentales, lo más importante es la dependencia psicológica, de ahí que la deshabituación psicológica se convierte en el aspecto esencial en ellas.

Conseguida la desintoxicación, o lo que es lo mismo, que la persona deje de consumir la sustancia, se pasa al proceso de deshabituación psicológica. Este es el proceso más largo y complejo del proceso adictivo, con la excepción de cuando la persona está en un programa de mantenimiento con metadona, en cuyo caso ambos procesos van en paralelo.

La deshabituación psicológica pretende conseguir que la persona dependiente de una sustancia psicoactiva o de una conducta adictiva sea capaz de afrontar la abstinencia. Por ello, en el caso de la dependencia de la heroína y de otras sustancias, se le entrena mediante distintas técnicas en afrontar la vida sin drogas, en poder evitarlas, en rechazarlas y en reorganizar su ambiente de modo que pueda estar sin ellas (Becoña y Vázquez, 2001). Esta es una de las partes más complejas de todo el proceso dado que las personas que acuden a tratamiento en ocasiones llevan un buen número de años consumiendo. Además, muchos han descubierto que cuando sienten malestar pueden ponerse mejor rápidamente consumiendo drogas. De ahí la relevancia que tiene el entrenamiento en estrategias de afrontamiento ante las situaciones de riesgo para el consumo. Las drogas producen efectos inmediatos. Entre el consumo y su efecto pasan pocos segundos. Hay 
también una gran disponibilidad de ellas y la persona sabe como acceder a las mismas. Entrenarlo adecuadamente en que adquiera habilidades para vivir sin drogas, que se encuentre bien subjetiva y anímicamente y que tenga apoyo en su ambiente, son algunas de las claves del éxito de un tratamiento. No se debe olvidar que las drogas consiguen no solo un efecto inmediato, sino que por el consumo previo se han hecho claramente reforzantes. Hay que buscar alternativas de refuerzo para el individuo y para que pueda contraponer las consecuencias negativas del consumo (ya que cuando es adicto solo ve las positivas) a las ventajas que tiene no consumir a nivel personal, familiar, social, etc.

Dado que sabemos que la recaída es algo íntimamente unido a la dependencia de cualquier sustancia, tanto sea de tipo legal como ilegal, o adicciones comportamentales, entrenarlo en prevenir la recaída es un elemento de gran relevancia una vez superadas las fases anteriores. Desde el modelo de Marlatt y Gordon (1985) y los desarrollos hechos a partir del mismo, con técnicas efectivas para que la persona no recaiga, la prevención de la recaída se ha convertido en un componente más del tratamiento. Con él podemos conseguir que la persona se mantenga abstinente y si recae pueda volver de nuevo a la abstinencia, o volver a la misma en el menor tiempo posible.

Finalmente, si la persona cambia su estilo de vida anterior por un estilo de vida saludable es más probable que mantenga la abstinencia a largo plazo. Aunque ello es hoy claro, la realidad nos muestra que un cambio en el estilo de vida no siempre es fácil, especialmente en los adictos a opiáceos, y va a depender de múltiples circunstancia, tanto del sujeto como de la familia y del medio social, oportunidades, madurez, etc. Cuando conseguimos un cambio en el estilo de vida relacionado con la abstinencia, entonces es más probable y fácil conseguir mantener la abstinencia tanto a corto como a largo plazo. El análisis de la comorbilidad, relacionado con el cambio en el estilo de vida, cobra una gran relevancia. Hacer un seguimiento 
Psicología de la salud y adiciones: perspectiva terapéutica

de ella y poder intervenir en los problemas asociados al consumo de drogas puede ser uno de los factores que facilitan el mantenimiento de la abstinencia.

El proceso de tratamiento de las adicciones: modelo general

A continuación exponemos un modelo general para el tratamiento de cualquier adicción. Con ello se puede también ver que las comunalidades entre las mismas permiten partir de un modelo general de tratamiento, para luego continuar con alguna técnica específica para adicciones concretas, como veremos en un punto posterior.

Las fases de un tratamiento para una persona con una adicción consta de las etapas de demanda del tratamiento, evaluación, tratamiento y seguimiento. A su vez, dentro del tratamiento, se distinguen seis fases: desintoxicación o mantenimiento; deshabituación psicológica o consecución de la abstinencia de la sustancia o dejar de hacer la conducta; normalización, cambio de estilo de vida anterior y búsqueda de nuevas metas alternativas a la adicción; prevención de recaídas; programa de mantenimiento o programa de apoyo a corto, medio y largo plazo; y, cuando sea necesario, programa de juego controlado o programas de reducción de daños (ej., en la dependencia de la heroína). Además, en éste como en otros trastornos, y por los problemas asociados al mismo, el psicólogo tiene que poner en marcha todos los recursos terapéuticos disponibles que nuestra ciencia nos proporciona.

En el Cuadro 1 se exponen con detalle dichas fases, el objetivo básico a conseguir en cada una de ellas, los elementos que debemos considerar y los procedimientos técnicos necesarios. Se exponen también las técnicas de tratamiento más importantes que se utilizan, prestando una atención especial a los aspectos motivacionales antes del tratamiento, a la prevención de la recaída y al mantenimiento a largo plazo. Las aplicaciones de este tratamiento pueden verse en Becoña (1996a, 1998a, 2001) y Becoña y Vázquez (2001). 


\section{Cuadro 1}

Esquema general para el tratamiento de una conducta adictiva

\section{Demanda de tratamiento}

Objetivo básico: analizar la demanda y retener al paciente en tratamiento.

Elementos a considerar: motivación para el cambio, estadios de cambio, recursos disponibles, apoyo de su medio, tratamientos previos, conocimientos de los tratamientos disponibles, tratamiento que desea, analizar quién está implicado en la demanda, por qué, etc.

Procedimientos técnicos: entrevista motivacional, escalas para evaluar aspectos motivacionales y estadios de cambio.

\section{Evaluación y análisis funcional}

Objetivo básico: conocer las áreas donde es más necesario e inmediato realizar cambios; conocer las áreas donde hay que realizar cambios a medio plazo; conocer las áreas donde los cambios a través del tratamiento y la reinserción social le pueden llevar a una vida normalizada. Con toda esa información disponible se realizará el análisis funcional de la conducta de juego y otras conductas asociadas al problema o problemas relacionados.

Elementos a considerar: evaluación individual, familiar, social, etc.

Procedimientos técnicos: entrevista clínica, autoinformes, cuestionarios, medidas psicofisiológicas, etc.

\section{Tratamiento}

Fase I. Desintoxicación o mantenimiento (ej., en el caso de la dependencia de la heroína)

Objetivo básico: desintoxicación física de la sustancia.

Elementos a considerar: tipo de desintoxicación en función de su petición, posibilidades asistenciales, recursos, etc., desde la ambulatoria a la hospitalaria.

Procedimientos técnicos: intervención médica frente a la hospitalaria para la consecución de la desintoxicación sin sufrir los síntomas del síndrome de abstinencia de los opiáceos.

Fase II. Deshabituación psicológica o consecución de la abstinencia de la sustancia o dejar de realizar la conducta

Objetivo básico: lograr la completa deshabituación psicológica a los indicios que conducen al paciente al consumo de la droga o a la realización de la conducta adictiva.

Elementos a considerar: adherencia al tratamiento, fase del tratamiento, aceptación del tratamiento propuesto, etc. 
Procedimientos técnicos: técnicas conductuales y cognitivas para la consecución de la abstinencia (por ejemplo, exposición con prevención de la respuesta, control de estímulos, entrenamiento en solución de problemas, entrenamiento en reducción del estrés, relajación, entrenamiento en habilidades sociales, técnicas de afrontamiento, reestructuración cognitiva, etc.). Se utilizará terapia individual, grupal y familiar.

Fase III. Normalización, cambio del estilo de vida anterior y búsqueda de nuevas metas alternativas a su conducta adictiva.

Objetivo básico: normalización en el sentido de ir adquiriendo más responsabilidades en el trabajo, en los estudios, en la familia, con los amigos, con la pareja, etc.; pago de las deudas; búsquéda de trabajo si no lo tiene, etc.

Elementos a considerar: grado de deterioro, si trabaja o no, si está o no separado, si padece enfermedades físicas (ej., VIH, hepatitis), etc.

Procedimientos técnicos: planificación de metas y objetivos, entrenarlo en adquirir nuevas habilidades, entrenamiento en asertividad, programa psicoeducativo, entrenamiento en solución de problemas, etc.

Fase IV. Prevención de recaídas

Objetivo básico: entrenarlo en técnicas de prevención de la recaída para que se mantenga abstinente a lo largo del tiempo.

Elementos a considerar: superación de las fases anteriores, mantenimiento de la abstinencia, efecto de la violación de la abstinencia.

Procedimientos técnicos: técnicas de prevención de recaídas (por ejemplo, autoinstrucciones, entrenamiento en solución de problemas, etc.).

Fase V. Programa de mantenimiento o programa de apoyo a corto, medio y largo plazo Objetivo básico: entrenarlo en estrategias de mantenimiento de la abstinencia a largo plazo y en cómo mejorar su calidad de vida.

Elementos a considerar: apoyo familiar, visitas de seguimiento a largo plazo, asistir a asociaciones de autoayuda, etc.

Procedimientos técnicos: entrenamiento en relajación, programación de actividades, procedimientos de manejo de estrés, etc.

Fase VI. Cuando sea necesario: programa de reducción de daños, programa de juego controlado, etc.

Objetivo básica: en el caso de la dependencia de la heroína reducir la prevalencia de VIH y mejorar los problemas causados por las drogas; en el juego patológico reducir la conducta de juego a un nivel donde no le acarree graves problemas.

Elementos a considerar: grado de deterioro de la persona, falta de adherencia al tratamiento psicológico, problemas psiquiátricos asociados, aceptación de este tipo de programa. 
Procedimientos técnicos: intervención mínima, programa psicoeducativo, educación para la salud y prácticas de seguridad ante la transmisión de enfermedades (VIH, hepatitis, etc.), técnica de control de estímulos, educación sobre el juego y las leyes de la probabilidad, educación a nivel familiar, laboral y de tiempo libre.

\section{Seguimiento}

Objetivo básico: conocer la evolución de la persona después del alta terapéutica. Elementos a considerar: modo de realizarlo (personalmente, en su casa, teléfono). Procedimientos técnicos: entrevista, autoinforme, urionoanálisis, etc.

Queda claro, inicialmente, que lo que se pretende es que la persona consiga la abstinencia. Este sigue siendo hoy el objetivo básico, aunque no se debe descartar una mejora en las condiciones de vida del individuo manteniendo la realización de la conducta a niveles bajos (ej., en el juego controlado o en los programas de reducción de daños o de sustitutivos opiáceos, como pasa con la metadona), pudiendo ser una meta desde el principio del tratamiento. De este modo se logra retener a la persona en tratamiento, reducir o eliminar su conducta problema y mejorar su funcionamiento cotidiano.

A continuación se exponen las distintas técnicas para los objetivos anteriores.

- Incremento de la motivación para el cambio. Hoy se sabe de la importancia que tiene la motivación para el cambio. En las conductas adictivas es frecuente que muchos de los pacientes no acudan a tratamiento. De los que acuden, una parte lo abandona después de la primera o primeras sesiones y, de los que acuden, no siempre siguen las instrucciones que se les dan. De ahí que en los últimos años este aspecto se haya convertido en un elemento terapéutico más, bajo la denominación de entrevista motivacional (Miller y Rollnick, 1999).

La entrevista motivacional, que se lleva a cabo en los primeros contactos entre el terapeuta y el paciente, es el modo más idóneo 
Psicología de la salud y adiciones: perspectiva terapéutica

para incrementar la motivación para el cambio. No hay que olvidar que no todas las personas con una adicción acuden la primera vez voluntariamente a tratamiento; más bien esto es la excepción. En ocasiones acuden por causas externas, bien sean de tipo familiar o legal. En otros casos, para buscar una ayuda puntual. Retener al sujeto en tratamiento, o más bien retenerlo al principio para que luego inicie un tratamiento, es una cuestión fundamental.

Muchas personas que acuden por primera vez a tratamiento piensan que no tienen un problema, que el problema es superficial o que aún no alcanzó el suficiente nivel de gravedad como para que haya que tratarlo. El objetivo de la primera entrevista, si ocurre esto, es convencerla de que sí tiene un problema, o de que alguien cercano a él lo tiene, y de que se le puede ayudar. Además, de poco sirve incluir a una persona en tratamiento si no quiere realizarlo o no está motivada para llevarlo a cabo. De hacerlo, probablemente el tratamiento va a ser inútil, lo va a abandonar a lo largo del mismo o no va a acudir a las siguientes sesiones.

La entrevista motivacional permite abordar la cuestión de la falta de motivación en aquellos sujetos en las fases de precontemplación (cuando no tienen ningún interés en cambiar su conducta) o contemplación (cuando tienen algún interés en cambiar su conducta pero no en este momento, sino en un futuro cercano). En ella se utilizan varias estrategias motivacionales, que han demostrado que son muy eficaces para motivar al paciente a cambiar su conducta. Estas serían ocho: dar información y aconsejar, eliminar obstáculos, dar diversas opciones al paciente para que pueda elegir, disminuir los factores que hacen que la conducta de consumo sea deseable, promover la empatía, dar retroalimentación, clarificar objetivos y proporcionarle ayuda activa. Con la entrevista motivacional podemos no sólo motivar al sujeto para que lleve a cabo el tratamiento sino a derribar las barreras y comenzar efectivamente el proceso de cambio.

- Exposición con prevención de la respuesta. La técnica de exposición con prevención de la respuesta es una técnica esencial en el 
tratamiento de los jugadores patológicos que acuden a tratamiento en España (Becoña, 1996b; Fernández-Montalvo y Echeburúa, 1997; González, Jiménez y Aymamí, 1999; Labrador y Fernández-Alba, 1998). Dado que el juego por el que acuden básicamente es por la adicción a las máquinas tragaperras o el bingo, esta técnica se convierte en central para conseguir dejar de jugar. Pero también es una técnica esencial en las otras adicciones (ej., dependencia de la heroína, adicción al sexo, etc.). A modo de ejemplo indicamos a continuación cómo se aplica a la adicción a las máquinas tragaperras.

El objetivo de la exposición es exponer al jugador a la máquina tragaperras para que mediante la presencia de los indicios que se han ido asociando a lo largo del tiempo con la máquina, note las sensaciones molestas que, sin prevención de la respuesta, o con dinero, le llevarían al juego. La prevención de la respuesta tiene como objetivo la imposibilidad de consumar su conducta, en este caso su conducta de jugar.

Los indicios más frecuentemente asociados a la conducta de juego de una persona con dependencia del juego en máquinas tragaperras es estar presente en el bar, mirar a la máquina, notar cómo las luces de colores de la máquina van cambiando en sentido hacia arriba, ver cómo otra persona introduce dinero en la máquina, comprobar que a un jugador le ha tocado un premio, cómo otras personas se fijan en la persona a la que le ha tocado un premio y comentan algo sobre ello, etc.

La exposición tiene que realizarse en vivo, bien acompañado por el terapeuta, un coterapeuta o bien realizar el propio jugador autoexposición. En este caso es conveniente que lo acompañe un familiar o persona de confianza. Cuando esto último no es posible tendría que autoexponerse por él mismo. Sin duda alguna, si se puede realizar alguna exposición con el terapeuta podemos controlar mejor dicho proceso, aunque es más costoso como procedimiento terapéutico. 
En los últimos años, a partir del programa de Ladouceur (Ladouceur, 1993; Ladouceur y Walker, 1998a, 1998b), se ha introducido también junto a la exposición con prevención de la respuesta el procedimiento de pensar en voz alta y la reestructuración cognitiva cuando la persona está jugando. Con ello se pretende no solo que la persona se exponga a la conducta de juego y no juegue sino que con ello cambie sus pensamientos y creencias irracionales por otros racionales, lo que le facilitaría a largo plazo no jugar y mantener así más fácilmente la abstinencia.

- Control de estímulos. Una técnica que se utiliza con frecuencia es la de control de estímulos, que en el caso del juego patológico es esencial para el tratamiento del individuo (Becoña, 1996b). En este caso, con ella restringimos el acceso a aquellos lugares en donde la probabilidad de jugar es mayor (calles donde hay ciertos bares con máquinas "calientes", calles donde hay bingos), u horas de mayor riesgo o de consumir una sustancia (ej., en la zona donde se "pilla" droga). Esto lo hacemos entrenando a la persona en la búsqueda de alternativas a sus anteriores costumbres o a que lo acompañe alguien en las situaciones que son de mayor riesgo para jugar o a comprar heroína.

Es determinante en este caso el control del dinero. Dado que el dinero es uno de los estímulos más importantes para que la persona juegue o compre la droga. Si no tiene dinero su probabilidad de jugar o comprar droga disminuye mucho. En este caso, el jugador tiene que transferir todo su dinero a algún familiar o a alguna persona cercana para que se lo controle y para que le dé solo el dinero que precisa para cada día. Además debe justificar todos los días, si fuer posible con tickets o facturas, en qué ha gastado el dinero para que no utilice parte de ese dinero en el juego (Fernández-Montalvo y Echeburúa, 1997; Labrador y Fernández-Alba, 1998).

En el caso de la dependencia a opiáceos esta técnica es muy útil en las primeras fases del tratamiento. Algunos de los estímulos más 
relevantes asociados al consumo son la conducta de búsqueda de drogas, amigos consumidores, lugares habituales de consumo, indicios ambientales, material para el consumo etc. Junto a esta restricción es importante que la persona sea capaz de generalizar dicho control para que en el futuro no recaiga.

- Entrenamiento en solución de problemas. El entrenamiento en solución de problemas es un procedimiento por el que se entrena a las personas a reconocer sus problemas, buscar soluciones adecuadas a los mismos e implantar la mejor solución en la situación donde ocurre el problema. Este entrenamiento consta de cinco fases: (a) orientación general hacia el problema; (b) definición y formulación del problema; (c) generación de soluciones alternativas; (d) toma de decisiones; y (e) puesta en práctica y verificación de la solución (D'Zurilla, 1986). En el entrenamiento en solución de problemas, para pasar de una fase a otra, hay que superar previamente la anterior. Cuando no hay suficiente información en una fase concreta, se ha infravalorado o saltado una fase previa, se está en una fase de entrenamiento, o la solución elegida no resulta la adecuada, hay que volver a la fase o fases previas para que una vez haya sido realizada correctamente podamos continuar con las siguientes.

En los últimos años el entrenamiento en solución de problemas se ha constituido en un elemento de muchos de los programas de tratamiento por su racionalidad, fácil de explicar al sujeto y su eficacia. Es, además, una estrategia incluida en casi todos los programas de prevención de la recaída.

- Terapia cognitiva. La terapia cognitiva parte de la premisa de que los trastornos se producen y mantienen debido a una serie de cogniciones subyacentes distorsionadas y a distintos errores en el procesamiento de la información. El tratamiento se orienta a corregir tanto esas premisas distorsionadas como los errores cognitivos (Beck, Wright, Newman y Liese, 1993). La importancia de las creencias y las urgencias son muy relevantes en la terapia cognitiva. Para Beck, a 
Psicología de la salud y adiciones: perspectiva terapéutica

partir del patrón cognitivo desadaptativo, ocurre la conducta (ej., el consumo) y lo biológico (ej., síndrome de abstinencia). Lo que la terapia cognitiva va a hacer es modificar los pensamientos y creencias erróneas del individuo y enseñarle técnicas de autocontrol. Aunque el planteamiento de la terapia cognitiva pueda parecer reduccionista, en la práctica no lo es. Junto al peso que se le da a la parte cognitiva de la persona, como causa de la explicación del consumo de sustancias, también se consideran como aspectos esenciales sus actuales problemas vitales, evolución desde la infancia, supuestos, estrategias compensatorias, elementos de vulnerabilidad, conducta, etc. De modo semejante, el tratamiento se va a centrar en varios de los aspectos que se relacionan con el problema (Beck et al., 1993). Se da gran relevancia a la relación terapéutica dentro del tratamiento y utiliza técnicas cognitivas como el diálogo socrático, la reatribución, las tareas para casa, identificar y modificar las creencias relacionadas con las drogas, relajación, solución de problemas, etc. Conforme avanza el tratamiento, junto al control de las urgencias y creencias asociadas a las mismas, cobra más relevancia la práctica de la activación de creencias de control, los otros problemas asociados a los problemas de consumo y la prevención de la recaída.

- Entrenamiento en reducción de la ansiedad y del estrés. Uno de los problemas asociados a la abstinencia del consumo de sustancias, como en el juego patológico, y otras adicciones, es la ansiedad y el estrés asociados a dicho estado. Por ello, es necesario evaluar esta problemática y aplicar técnicas para su control. Los programas específicos pretenden alterar la percepción del grado de amenaza que se le atribuye al estresor, su estilo de vida para reducir tanto la frecuencia como la severidad de los estresores externos y capacitarles para usar estrategias de afrontamiento activas que inhiban o reemplacen las respuestas de estrés incapacitantes.

Como técnica de intervención general se puede utilizar cualquiera de las existentes para este problema, como por ejemplo, el entre- 
namiento en manejo del estrés y las técnicas de relajación, las técnicas cognitivas, la biblioterapia, el cambio del estilo de vida, etc. En la práctica el entrenamiento en relajación es una técnica ampliamente utilizada junto a las de tipo cognitivo, para cambiar las creencias erróneas sobre las causas de la ansiedad o de los elementos estresantes. Cuando padece un trastorno de ansiedad específico (por ejemplo, ataques de pánico) es necesario aplicar aquellas técnicas específicas que son eficaces para el tratamiento del mismo (Gutiérrez, 2003).

- Entrenamiento en habilidades sociales. Muchas personas con dependencia de distintas drogas y en otras adicciones comportamentales se les proporciona entrenamiento en habilidades sociales para mejorar su competencia social. Cuando carecen de habilidades interpersonales e intrapersonales adecuadas, de habilidades para controlar su estado emocional sin acudir a jugar o a consumir la sustancia y de habilidades para manejar su relación de pareja, con los hijos, en el trabajo, etc., este entrenamiento es imprescindible. El entrenamiento en habilidades sociales se convierte de este modo en una parte importante del tratamiento cuando hay un déficit en las mismas (Monti, Rohsenow, Colby, y Abrams, 1995). Además, con ello se conseguirá disponer de una estrategia de prevención de la recaída para el futuro. Las situaciones de recaída se dan cuando existe frustración e incapacidad de expresar ira, incapacidad de resistir la presión social, estado emocional negativo intrapersonal, incapacidad de resistir la tentación intrapersonal, entre otras.

La potencial relación entre las pobres habilidades sociales y las actividades de juego o consumo de drogas es discutida con los pacientes. Es importante reconocer que las personas con adicciones pueden necesitar más que las habituales habilidades sociales para afrontar sus conflictos relacionales. Por ejemplo, algunos jugadores necesitan entrenamiento en asertividad para mejorar su habilidad para rechazar las invitaciones para jugar con sus amigos. El juego de roles puede ser utilizado para mejorar las habilidades de comunicación. Este entrenamiento se focaliza sobre las consecuencias negativas de 
Psicología de la salud y adiciones: perspectiva terapéutica

jugar y cómo la carencia de adecuadas habilidades sociales es un factor que contribuye a ello (Becoña, 1996a).

- Control de la ira y de la agresividad. En ocasiones, la persona con dependencia de sustancias tiene problemas asociados de ira y agresividad. Los mismos, a su vez, le acarrean distintos problemas en su medio familiar, social o policial. Si esto ocurre pueden incrementar aún más su problema inicial de dependencia o mantenerse su dependencia en el tiempo por no tener un modo de solucionar el otro problema. También, tanto la ira como la agresividad, están asociadas a la caída y a la recaída, como estados emocionales negativos (Marlatt, Barrett y Daley, 1999).

Cuando este es el caso es necesario utilizar estrategias de control de la ira y de la agresividad tal como la técnica de inoculación de estrés, juego de roles, entrenamiento asertivo, entrenamiento en relajación, solución de problema o reestructuración cognitiva.

- Manejo de contingencias. El consumo de drogas se mantiene por el reforzamiento positivo que la sustancia produce en la persona (por ej., euforia, placer) y por el reforzamiento negativo (consumo para evitar las consecuencias negativas del síndrome de abstinencia). El consumo de drogas, por tanto, está influenciado por el aprendizaje y por el condicionamiento. También, mediante los principios del aprendizaje y del condicionamiento podemos cambiar esta conducta desadaptada por otra adaptada sin consumo de drogas. Esto se hace mediante los principios básicos del manejo de contingencias, el reforzamiento positivo y negativo, el castigo positivo y negativo y las distintas técnicas derivadas de los mismos (por ej., extinción o control de estímulos) (Graña, 1994).

Desde la perspectiva operante se sabe que el desarrollo de un trastorno por abuso o dependencia de sustancias se debe tanto al poder reforzante de la droga como de otros factores, como los biológicos, ambientales y variables conductuales (Becoña, 1999). Pero, sin negar 
esto, de cara al tratamiento, se propone un mecanismo de intervención, la alteración de las contingencias conductuales, que es independiente de los factores etiológicos específicos.

Técnicas como la aplicación de estímulos aversivos, reforzar conductas alternativas incompatibles con consumir drogas, extinción o varias de las anteriores al mismo tiempo, facilitan el cambio de la conducta de consumo. Otra extensión de las mismas son los contratos de contingencias o las estrategias de prevención de recaídas. La utilización de técnicas de manejo de contingencias, muchas veces, junto a otras técnicas, nos permite tener un abordaje útil y eficaz para muchos pacientes que acuden a tratamiento. Especialmente para que una persona lleve a cabo conductas alternativas al consumo de drogas, el manejo de contingencias a veces es la técnica principal para que la persona mantenga su abstinencia. Alguna técnica ya vista, como la de control de estímulos, son técnicas de control de contingencias que tienen una gran relevancia tanto para el tratamiento de las personas con dependencia de la heroína como del resto de las conductas adictivas.

- Técnicas de autocontrol. Una de las estrategias en el tratamiento de las distintas adicciones, tanto en un programa orientado a la abstinencia como al jugar controlado o a la reducción de daños es entrenarlos en técnicas de autocontrol (Hester, 1995). Esto puede hacerse una vez que la persona ha aceptado participar en un tratamiento orientado a la abstinencia, cuando tiene dificultades en conseguirla o cuando su objetivo es el juego controlado o la reducción de daños. En ocasiones el autocontrol se pone en marcha en la parte media del tratamiento cuando ya se han conseguido los objetivos mínimos para que mantenga la adherencia al tratamiento.

Las técnicas de autocontrol se orientan a que la persona conozca su conducta problema y sea capaz de afrontar la misma mediante técnicas que ha aprendido sin llevarla a la práctica. Con el autocontrol 
Psicología de la salud y adiciones: perspectiva terapéutica

se enseña a la persona estrategias para controlar o modificar su propia conducta a través de distintas situaciones, con el propósito de alcanzar metas a largo plazo.

Para ello se entrena a la persona en distintas técnicas para que no realice la conducta o, en caso de que se produzca la conducta, que lo haga de un modo poco problemático y con la idea de poder llegar a conseguir la abstinencia total de la misma.

El terapeuta le ayuda a establecer condiciones favorables para el cambio, le entrena en técnicas para conseguirlo y le refuerza por los avances conseguidos. También le entrena en la aplicación de autorefuerzos, en buscar apoyo en otras personas para mantener las ganancias conseguidas y que las personas de su ambiente le refuercen su abstinencia. Existen una serie de técnicas que se utilizan sistemáticamente cuando se aplica un programa de autocontrol (ej., autoregistros, cambio de estímulos discriminativos, técnicas encubiertas). Para el buen diseño del mismo hay que hacer una evaluación conductual adecuada del problema que tiene la persona y adaptar el tratamiento a los avances que se van produciendo a lo largo del mismo y a la consecución de los objetivos iniciales e intermedios. También, las técnicas de autocontrol dan una gran importancia al mantenimiento de los cambios, especialmente a través de la utilización del autorefuerzo, cuando la conducta la realiza en la dirección esperada, y el autocastigo cuando se lleva a cabo la conducta indeseada. Finalmente, cada vez cobran más importancia los procedimientos cognitivos dentro del autocontrol, especialmente por las ideas, creencias, pensamientos o atribuciones erróneas que la persona tiene sobre su juego y el ambiente de juego, así como sobre el resultado del mismo.

- El tratamiento de los problemas de comorbilidad. Aproximadamente el $90 \%$ de las personas con dependencia de opiáceos tiene un diagnóstico psiquiátrico adicional y el $15 \%$ intentan suicidarse al menos una vez. No menos de un $25 \%$ de las personas jugadoras patoló- 
gicas van a tener un problema de depresión mayor y de un 15 a un $20 \%$, problemas de dependencia del alcohol. También, otros problemas como trastornos de ansiedad, trastornos de personalidad, u otros, pueden estar presentes (Grant y Dawson, 1999). De ahí que diagnosticar y tratar estos trastornos asociados es una parte integral del tratamiento.

Cuando se detectan este tipo de problemas, se abordarán con intervenciones específicas de tipo cognitivo-conductual, farmacológico o una combinación de ambas modalidades, para cada uno de los trastornos y adaptadas a las necesidades específicas del paciente. Uno de los problemas que van a surgir entonces es si los tratamientos se realizarán de forma integrada por un mismo equipo terapéutico (modelo integracionista), si se debe iniciar el tratamiento de un trastorno y posteriormente el del otro (modelo secuencial) o si, por el contrario, se deben abordar a la vez. En los últimos años se ha hecho hincapié que para el tratamiento de los trastornos duales se debe adoptar un modelo integracionista. Esto significa que los tratamientos de ambos trastornos se deben hacer de modo simultáneo y por el mismo equipo terapéutico.

- El tratamiento de los problemas maritales y familiares: terapia de pareja y terapia familiar. Las conductas adictivas inciden de modo muy acusado en la vida de la persona en su familia o pareja. Esta es una de las áreas más afectadas, encontrándose en ocasiones sus personas más queridas «presas» de su proceso adictivo. Si éste se ha mantenido en el tiempo ellos han sufrido directamente el desapego, el deterioro físico, los problemas con sus amigos, con la justicia, etc., de ese miembro de la familia o pareja.

Una vez que ha entrado en tratamiento, es necesario intervenir en la pareja, si es el caso, con terapia de pareja. Y, si la familia nuclear o más extensa es la afectada, mediante terapia familiar (Heath y Stanton, 1998). La terapia de pareja es un tipo de terapia específica 
Psicología de la salud y adiciones: perspectiva terapéutica

que pretende incrementar la comunicación entre ambos, conseguir un incremento en el intercambio de refuerzos entre los dos miembros de la pareja y solucionar entre ellos los problemas que les vayan surgiendo, entrenándoles en técnicas de solución de problemas. También es necesario entrenarlos en aquellos otros aspectos que les inciden en dicha relación (por ejemplo, manejo de los hijos, problemas sexuales de uno de los miembros, etc.).

La terapia familiar tiene su aplicación cuando hay una afectación de toda la familia ante un miembro problema (la persona con adicción) y por las relaciones peculiares que se establecen entre el adicto y uno o más miembros de la familia. Es aplicable cuando el adicto suele ser joven y vive con su familia. La terapia familiar suele partir mayoritariamente del concepto de sistema, entendiendo que las experiencias y conductas de una persona están asociadas y dependen de la conducta de otros miembros de la familia. Esto es, la conducta de un miembro no se puede entender aislada de la de los demás. Cada familia desarrolla patrones de comunicación y secuencias de conductas para mantener el equilibrio entre sus miembros. La terapia familiar conductual lo que va a hacer es entrenar a los miembros de la familia en técnicas de manejo de contingencias (por ejemplo, contratos conductuales), entrenamiento en habilidades de solución de problemas y de comunicación, intercambio de conductas, observación de la propia conducta y la de los demás, intercambio de refuerzos entre los distintos miembros de la familia, cambio de expectativas y atribuciones negativas, reducir los estados emocionales negativos. Especialmente, cuando la persona consigue la abstinencia o deja de hacer la conducta adictiva, hay que estar atento a posibles cambios en la estructura relacional de la familia que puede facilitar la recaída del mismo. Tener comprometida a la familia en el tratamiento puede evitar lo anterior, junto a un adecuado manejo de los conflictos subyacentes o manifiestos en la familia y disponer de adecuadas habilidades de comunicación y de solución de problemas. Los problemas que hay que manejar adecuadamente para poder lograr todo lo 
anterior son tener unas claras normas de convivencia, de jerarquía y de comunicación dentro de la familia y que el padre se implique en la familia y en el problema que tiene su hijo y no deje todo el peso en la madre, desentendiéndose del problema. De ahí que, en algunos casos, la intervención se hace más amplia y compleja de lo que puede parecer en un principio cuando otros miembros distintos al individuo, como es en este caso la familia, facilitan o pueden mantener en parte su problema. Por ello, en ocasiones, la terapia familiar se lleva a cabo solo con los padres o en grupos de padres, para entrenar a éstos en el manejo adecuado de sus hijos y para que tengan una adecuada implicación emocional con ellos y un comportamiento que facilite la recuperación de su hijo o hija (Martínez, 2003).

- Terapia de grupo. La terapia de grupo es una modalidad terapéutica que se utiliza con frecuencia para el tratamiento de las personas con distintas adicciones (Galanter, Castañeda y Franco, 1998). Junto al tratamiento individual, y las otras intervenciones que pueda estar llevando a cabo, la terapia de grupo es de gran relevancia en varios de los tratamientos específicos para las adicciones, especialmente quizás en las personas que acuden a los centros de drogodependencias y a las asociaciones de autoayuda, en donde la misma es una parte esencial del tratamiento.

El objetivo que se pretende con la terapia de grupo es el mismo que el que se pretende con la terapia individual pero en un formato de grupo y con las dinámicas propias que se van desarrollando dentro de cada grupo. Le permite al paciente compararse con otros, tener apoyo, aprender estrategias y técnicas de control, adquirir habilidades y asumir normas de funcionamiento del grupo. Los objetivos centrales del mismo son solucionar problemas y realizar tareas que le lleven a la abstinencia o le permita mantener la misma para, finalmente, poder cambiar su estilo de vida.

- Prevención de la recaída. El primer consumo de la sustancia o tener un episodio de juego después de dejarlo no tiene por qué repre- 
Psicología de la salud y adiciones: perspectiva terapéutica

sentar una recaída, puede ser sólo una caída o un desliz puntual. Para Marlatt y Gordon (1985) la recaída se define como cualquier retorno al comportamiento adictivo o problemático o al estilo de vida anterior, después de un período inicial de abstinencia y de cambio en el estilo de vida. Una caída es un retorno breve a la conducta adictiva en un momento concreto del tiempo, es decir, una pérdida de control puntual sobre la conducta, que puede llevar a la persona a realizar algún consumo esporádico. La recaída en las adicciones se puede manifestar de distintas formas: volver al estilo de vida anterior, consumir drogas sustitutivas, realizar actividades de azar y de riesgo o sexualidad compulsiva, etc. En el caso del juego la forma más común de manifestarse es volver a jugar de forma regular. Lo mismo ocurre en el caso de las sustancias psicoactivas.

Un concepto fundamental para comprender el proceso de recaída es el llamado efecto de violación de la abstinencia. Este efecto se compone de dos elementos cognitivos clave: la disonancia cognitiva (conflicto y culpa) y un efecto de atribución personal (culparse a uno mismo como causa de la recaída), así como algunas veces la anticipación de los efectos positivos en relación a la droga o al juego. La combinación de estos tres componentes predisponen al paciente a una recaída total.

El modelo de prevención de la recaída considera a las adicciones como un hábito adquirido que puede cambiarse aplicando los principios del condicionamiento clásico, operante y vicario. Además da una gran importancia a los factores cognitivos implicados en la recaída. Las estrategias de prevención de la recaída se orientan a anticipar y prevenir la ocurrencia de recaídas después del tratamiento y a cómo ayudar a los pacientes a afrontar la recaída, si se produce. Es un programa de autocontrol donde se enseña a los pacientes a anticipar y a afrontar eficazmente los problemas que aparezcan después del tratamiento o en el seguimiento. Por ello, la prevención de la recaída se puede aplicar como estrategia de mantenimiento, para preve- 
nir la recaída o con un enfoque más general, para cambiar el estilo de vida.

Las causas principales de la recaída en las adicciones son los estados emocionales negativos, conflicto interpersonal y presión social (ej., Marlatt y Gordon, 1985). Los estados emocionales negativos son situaciones en las que los sujetos experimentan un estado emocional, estado de ánimo o sentimientos negativos, como frustración, ira, ansiedad, depresión o aburrimiento, antes o al mismo tiempo que la ocurrencia de la primera caída. Los conflictos interpersonales son situaciones que incluyen un conflicto actual o relativamente reciente, asociado con cualquier relación interpersonal, como el matrimonio, los miembros de la familia, relaciones en el trabajo, etc. La presión social se refiere a situaciones en las que el paciente responde a la influencia de otra u otras personas que ejercen presión para involucrarlo de nuevo en el consumo de sustancias.

Si la persona es capaz de ejecutar una respuesta de afrontamiento eficaz en una situación de alto riesgo, la probabilidad de recaída disminuye. Al afrontar exitosamente una situación de alto riesgo es probable que la persona experimente una sensación de dominio y percepción de control, generándose una expectativa de ser capaz de afrontar eficazmente otras situaciones de riesgo que se le vayan presentando (autoeficacia). Si esto se repite adquirirá una mayor percepción de control y disminuirá la probabilidad de recaída. Si, en cambio, no es capaz de afrontar con eficacia una situación de alto riesgo y si tiene una expectativa positiva de volver a consumir la droga o de volver a realizar la conducta adictiva, aumenta la probabilidad de recaída.

Hay tres principales estrategias para utilizar en los procesos de prevención de la recaída: la aproximación de apoyo social, la aproximación de cambio de estilo de vida y la aproximación cognitiva conductual (Becoña, 1999). Las técnicas cognitivo-conductuales para la prevención de la recaída son las más eficaces. Se orientan a aumentar la 
autoeficacia, mejorar el control de impulsos, favorecer la reestructuración cognitiva y mejorar las estrategias de toma de decisiones. Las estrategias dirigidas al cambio en el estilo de vida están dirigidas a fortalecer la capacidad global de afrontamiento del paciente y a reducir la frecuencia e intensidad de los impulsos y deseos irresistibles de jugar que a menudo son el producto de un estilo de vida desequilibrado. Dentro de las técnicas de prevención de la recaída que se aplican están los programas de reestructuración cognitiva, el entrenamiento en identificación y control de estímulos, exposición a estímulos en vivo (reales) o a nivel imaginado, detención del pensamiento, condicionamiento encubierto, programas de refuerzo externo, etc. Si en una situación de alto riesgo el paciente anticipa lo que le puede ocurrir (por ejemplo, cuando pasa por delante del bar en el que habitualmente jugaba o ante el barrio en el que adquiría la sustancia) y tiene una sensación extraña semejante a cuando entraba y jugaba o consumía la droga, puede poner en marcha distintas estrategias para afrontarla, especialmente cuando tiene urgencias, para así poder hacerle frente y no caer o recaer de nuevo en su problema adictivo.

\section{Perspectivas}

Las conductas adictivas se han convertido en estos años en un grupo de trastornos de gran relevancia. Tal relevancia viene motivada por su alta prevalencia en las personas, tanto sean adultas, como adolescentes. El número de personas con dependencia de la nicotina y del alcohol, solamente, nos lleva a contar el número de personas en un país como el nuestro en millones (Becoña y Vázquez, 1998). Otras, aunque lo son en menor grado, como la adicción a drogas ilegales, ocupan un lugar nada despreciable, especialmente por los problemas asociados que acarrea su consumo, especialmente en los últimos años en la esfera física, aparte de la gran alarma social que han producido en las últimas décadas el consumo de drogas como la heroína, la cocaína y la marihuana, y todavía sigue produciendo en ésta y en nuevas sustancias (ej., drogas de síntesis). Otras adicciones, 
las comportamentales, como el juego patológico, las compras compulsivas, el sexo compulsivo, la adicción a internet y otras, son adicciones que van en paralelo al desarrollo de las sociedades industrializadas, al ocio y tiempo libre (Calafat et al., 2001) y a una nueva forma de vida, en comparación a lo que ocurría hace no más de 50 años.

El tratamiento de las adicciones ha cobrado una enorme relevancia en las últimas décadas. Con la interrupción de las drogas ilegales, especialmente heroína y cocaína, en los países desarrollados, desde los años 70 hasta el momento presente, la demanda de tratamiento no ha dejado de crecer y los problemas causados por las mismas son hoy de una gran relevancia. Además, con el surgimiento del VIH y el SIDA unida a las mismas ha producido un vuelco inesperado a esta problemática y a un incremento del interés, de los medios y de los recursos para atajar tanto la epidemia del SIDA como los problemas de drogadicción. Todo ello ha producido el surgimiento de una nueva área de trabajo e investigación que no ha dejado de crecer. El problema está en que el problema se mantiene en un nivel estable, o en fase de crecimiento en algunos países, cada vez más tenemos que hablar de un trastorno crónico o caracterizado por las frecuentes recaídas, lo que lleva a tener que plantear programas de tratamiento complejos y a largo plazo (Becoña y Vázquez, 2001). Realmente el mejor modo de atajar este problema es a través de la prevención (Becoña, 1999), pero en nuestra sociedad no hay una idea preventiva bien asentada y los recursos suelen proporcionarse al tratamiento y a la rehabilitación, ya una vez que el problema existe. El incidir en actividades de tipo preventivo, como caracteriza a la psicología de la salud, es un adecuado abordaje para este problema (Oblitas y Becoña, 2000).

El éxito del abordaje psicológico de las adicciones ha estado en que el mecanismo explicativo es primeramente social, en segundo lugar psicológico y en menor grado, biológico (Becoña, 1999). Esto no significa que unos elementos sean menos relevantes que otros, pero 
Psicología de la salud y adiciones: perspectiva terapéutica

sí que ésta es su graduación. Como sobre el elemento social es difícil intervenir, porque corresponde al desarrollo natural de las sociedades, modulado por su sistema cultural previo, económico y político actual, la intervención sobre la conducta, sobre la conducta del individuo en su contexto más cercano, se ha convertido en el campo de trabajo más relevante para ayudar especialmente a las personas que tienen problemas de adicción a sustancias y de adicción a conductas. Además, en el caso de la adicción a conductas, la mejor explicación es la psicológica, aunque, insistimos, desde una explicación macro, el entorno social, la cultura y el tipo de vida actual en muchas ocasiones están en la base del trastorno. Aunque es indudable que el componente biológico existe y que sin él no habría adicciones, la evolución del individuo a lo largo de la historia evolutiva y genética nos permite a la mayoría de nosotros ser capaces de afrontar adecuadamente las distintas adicciones y superarlas, aún en caso de que tuviésemos contacto con ellas. De ahí que tener en cuenta una visión comprensiva y global de este tipo de conductas es muy importante para poder ayudar más efectivamente a las personas que acuden a nosotros en busca de ayuda y para que les podamos ayudar de modo adecuado y eficaz (Becoña, 1999).

Por suerte, en los últimos años se han hecho grandes esfuerzos y buen número de investigaciones para conocer mejor este tipo de conductas, bien de modo general (adicciones), o específicamente para cada una de ellas (ej., alcoholismo, tabaquismo, dependencia de la heroína, juego, etc.). Ello ha favorecido el campo del tratamiento, en donde también se han dado pasos muy importantes en las áltimas décadas. Ello ha permitido y favorecido el que hayan surgido o se hayan aplicado, distintos tratamientos psicológicos y/o farmacológicos, muchos de ellos con una buena evaluación y eficacia (Labrador, Echeburúa y Becoña, 2000). Dentro de los tratamientos psicológicos han destacado los tratamientos cognitivo-conductuales que son el tratamiento de elección para las distintas conductas adictivas, bien en exclusiva (ej., en el tratamiento del juego patológico, adicción al 
sexo o internet, cocaína, etc.) o como una parte básica del mismo (ej., dependencia de la heroína, alcoholismo, etc.). Y esto ha sido debido tanto por la eficacia del tratamiento psicológico de tipo cognitivo-conductual como por su racionalidad y escaso tiempo de intervención, en comparación con otros abordajes terapéuticos. Pero aún así, hay que saber que en ocasiones el tratamiento puede ser largo y lleno de avatares, porque la recaída es una de sus características definitorias. $Y$, de lo que no tenemos hoy dudas es que las conductas adictivas, como hemos insistido (Becoña, 1998b), van a mantenerse en el futuro -siendo optimistas- o lo más probable es que el actual nivel de prevalencia se incremente aún más. De ahí la necesidad de disponer cada vez más de abordajes terapéuticos efectivos, que estén disponibles para los afectados y convencerlos de que acudan a tratamiento lo antes posible para evitar la cronificación del trastorno y para evitar que cuando acudan a tratamiento el abordaje terapéutico sea más complejo y la recuperación más difícil.

Finalmente, como afirmábamos en otro estudio (Becoña, 1998b), consideramos que las conductas adictivas serán el gran problema del siglo XXI, a nivel cuantitativo, porque los datos que tenemos a nivel epidemiológico de personas dependientes del alcohol, del tabaco, de las drogas ilegales y de las adicciones comportamentales, nos muestran que un porcentaje muy significativo de la población, que puede ser a veces de un tercio o un cuarto de la misma tenga -según los criterios diagnósticos actuales- un problema de dependencia a una adicción (ej., el porcentaje de personas dependientes de la nicotina y del alcohol en nuestro país). El que no sea más relevante este problema y no se dediquen más medios al mismo está en los intereses, los enormes intereses económicos, que subyacen a la mayoría de estas conductas adictivas, que mueven diariamente muchos cientos de millones de dólares. 


\section{Conclusiones}

Las conductas adictivas, sean con sustancia o sean comportamentales, constituyen actualmente un problema de gran relevancia social y clínica en las sociedades desarrolladas. Apenas existentes hace unas décadas, en el momento actual tienen un nivel de prevalencia muy alto en la población. Ello ha llevado al desarrollo de técnicas específicas de tratamiento para ellas, interés por parte de los profesionales, y disponer de recursos importantes para el tratamiento. En los próximos años este interés se mantendrá y puede que se incremente por la cronificación del trastornos en muchas de las personas que lo padecen, por las consecuencias colaterales que puede producir (ej., SIDA) y por la necesidad de atajar un problema que es epidemiológicamente -desde la perspectiva clínica- el más relevante que tenemos actualmente. Por suerte, disponemos de adecuados tratamientos para estos trastornos, aunque es necesario seguir perfeccionándolos para que de ese modo podamos ayudar más efectivamente a este tipo de personas.

\section{Referencias}

American Psychiatric Association (1994). Diagnostic and statistical manual of mental disorders (4ta. ed.). Washington, DC: autor.

Beck, A. T., Wright, F. D., Newman, C. F. y Liese, B. S. (1993). Cognitive therapy of substance abuse. Nueva York: Guildford Press. Becoña, E. (1996a). La ludopatía. Madrid: Aguilar.

Becoña, E. (1996b). Tratamiento del juego patológico. En J. M. Buceta y A. M. Bueno (Eds.), Tratamiento psicológico de hábitos y enfermedades (pp. 249-278). Madrid: Pirámide.

Becoña, E. (1998a). Alcoholismo. En M. A. Vallejo (Ed.), Manual de terapia de conducta (vol. 2, pp. 75-141). Madrid: Dykinson.

Becoña, E. (1998b). Conductas adictivas. ¿El problema del siglo XXI? Psicología Contemporánea, 5, 4-15.

Becoña, E. (1999). Bases teóricas que sustentan los programas de prevención de drogas. Madrid: Plan Nacional sobre Drogas. 
Becoña, E. (2001). Tratamiento psicológico de las conductas adictivas. En J. M. Buceta, A. M. Bueno y B. Mas (Eds.), Intervención psicológica y salud: Control del estrés y conductas de riesgo (pp. 423-462). Madrid: Dykinson.

Becoña, E. y Vázquez, F. L. (1998). Tratamiento del tabaquismo. Madrid: Dykinson.

Becoña, E. y Vázquez, F.L. (2001). Heroína, cocaína y drogas de sintesis. Madrid: Síntesis.

Calafat, A., Juan, M., Becoña, E., Fernández, C., Gil, E., Palmer, A., Sureda, P. y Torres, M. A. (2000). Salir de marcha y consumo de drogas. Madrid: Plan Nacional sobre Drogas.

D"Zurilla, T. J. (1986). Problem-solving therapy. A social competence approach to clinical interventions. Nueva York, NY: Springer.

Echeburúa, E. (1999). ¿Adicciones sin drogas? Bilbao: Desclée de Brouwer.

Fernández-Montalvo, J. y Echeburúa, E. (1997). Manual práctico del juego patológico. Ayuda para el paciente y guía para el terapeuta. Madrid: Pirámide.

Galanter, M., Castañeda, R. y Franco, H. (1998). Group therapy, selfhelp groups, and network therapy. En R. J. Frances y S. I. Miller (Eds.), Clinical textbook of addictive disorders (2da. ed.) (pp. 521-546). Nueva York: Guildford Press.

González, A., Jiménez, S. y Aymamí, M.N. (1999). Evaluación y tratamiento cognitivo-conductual de jugadores patológicos de máquinas tragaperras con premio. Anuario de Psicología, 30, 111125.

Gossop, M. (Ed.) (1989). Relapse and addictive behaviour. Londres: Routledge.

Grant, B. F. y Dawson, D. A. (1999). Alcohol and drug use, abuse, and dependence: classification, prevalence, and comorbidity. En B. S. McCrady y E. E. Epstein (Eds.), Addictions. A comprehensive guidebook (pp. 9-29). Nueva York, NY: Oxford University Press.

Graña, J. L. (1994). Intervención conductual individual en drogodependencias. En J. L. Graña (Ed.), Conductas adictivas. Teoría, evaluación y tratamiento (pp. 141-190). Madrid: Debate. 
Psicología de la salud y adiciones: perspectiva terapéutica

Gutiérrez, J. (2003). Ansiedad y Salud. En L.A. Oblitas (Ed.), Psicología de la salud y la calidad de vida. México: Thomson (en prensa).

Heath, A. W. y Stanton, M. D. (1998). Family-based treatment: States and outcomes. En R. J. Frances y S. I. Miller (Eds.), Clinical textbook of addictive disorders (2da. ed.) (pp. 496-520). Nueva York: Guildford Press.

Hester, R. K. (1995). Behavioral self-control training. En R. K. Hester y W. R. Miller (Eds.), Handbook of alcoholism treatment approaches. Effective alternatives (2da. ed.) (pp. 148-159). Boston, MA: Allyn and Bacon.

Labrador, F. J., Echeburúa, E. y Becoña, E. (2000). Guía para la elección de tratamientos psicológicos efectivos. Hacia una nueva psicología clínica. Madrid: Dykinson.

Labrador, F. J. y Fernández-Alba, A. (1998). Juego patológico. En M. A. Vallejo (Ed.), Manual de terapia de conducta (Vol. 2) (pp. 143-211). Madrid: Dikynson.

Ladouceur, R. (1993). Aspectos fundamentales y clínicos de la psicología de los juegos de azar. Psicología Conductual, I, 361-374.

Ladouceur, R. y Walker, M. (1998a). Aproximación cognitiva para la comprensión y tratamiento del juego patológico. Psicología Contemporánea, 5, 56-71.

Ladouceur, R. y Walker, M. (1998b). Cognitive approach to understanding and treating pathological gambling. En P. Salkovskis (Ed.), Comprehensive clinical psychology. Adults: Clinical formulation \& treatment (Vol. 6) (pp. 587-601). Oxford, RU: Elsevier Science.

Marlatt, G. A. y Gordon, J. (1985). Relapse prevention. Maintenance strategies in addictive behavior change. Nueva York: Guildford Press.

Marlatt, G. A., Barrett, K. y Daley, D. C. (1999). Relapse prevention. En M. Galanter y H. D. Keller (Eds.), Textbook of substance abuse treatment (2da. ed.) (pp. 353-366). Washington, DC: The American Psychiatric Press. 
Martínez, A. (2003). Terapia de familia. En L.A. Oblitas (Ed.), 16 enfoques psicoterapéuticos contemporáneos. Bogotá: Psicología científica (en prensa).

Miller, W. R. y Rollnick, S. (1999). La entrevista motivacional. Preparar para el cambio de conductas adictivas. Barcelona: Paidós. Monti, P. M., Rohsenow, D. J., Colby, S. M. y Abrams, D. B. (1995). Coping and social skills training. En R. K. Hester y W. R. Miller (Eds.), Handbook of alcoholism treatment approaches. Effective alternatives (2da. ed.) (pp. 221-241). Boston, MA: Allyn and Bacon.

Oblitas, L. y Becoña, E. (Eds.) (2000). Psicología de la salud. México: Plaza y Valdés.

Westermeyer, J. (1998). Historial and social context of psychoactive substance disorders. En R. J. Frances y S. I. Miller (Eds.), Clinical textbook of addictive disorders (2da. ed.) (pp. 14-32). Nueva York: Guildford Press. 